\title{
Effects of obesity and weight loss on microRNA expression in the human colorectal mucosa
}

\author{
$\underline{\text { Stella Breininger }}^{1,2}$, Laura Sabater ${ }^{3}$, Fiona Malcomson ${ }^{1}$, Sorena Afshar ${ }^{1}$, Jelena Mann ${ }^{3}$ and \\ John Mathers ${ }^{1}$ \\ ${ }^{1}$ Human Nutrition Research Centre, Institute of Cellular Medicine and LLHW Centre for Ageing and Vitality, \\ Newcastle University, Newcastle upon Tyne, United Kingdom, \\ ${ }^{2}$ Wellcome Trust Centre for Mitochondrial Research, Newcastle University, Newcastle upon Tyne, United Kingdom and \\ ${ }^{3}$ Fibrosis Research Group, Newcastle University, Newcastle upon Tyne, United Kingdom
}

\begin{abstract}
Introduction: Colorectal cancer $(\mathrm{CRC})$ is the $3^{\text {rd }}$ most common cancer worldwide. Obesity, and its lifestyle determinants, physical inactivity and poor diet, increase CRC risk. However, the effects of weight loss by bariatric surgery on CRC risk are unclear. Epigenetic mechanisms involving microRNAs that lead to dysregulated gene expression may mediate the effects of obesity and weight loss on CRC risk. We hypothesised that microRNAs are i) aberrantly expressed in obese individuals compared with healthy non-obese individuals and ii) modulated by significant weight loss following bariatric surgery.
\end{abstract}

Methods: We used data and samples from the Biomarkers of Colorectal Cancer after Bariatric Surgery (BOCABS) Study. Obese patients listed for bariatric surgery and age- and sex-matched healthy non-obese adults (Controls) were recruited at North Tyneside General Hospital. Rectal mucosal biopsies were collected at baseline and six months post-surgery from obese participants and at baseline only from Controls. Using Next Generation Sequencing and bioinformatics analysis, a panel of 8 microRNAs was selected and validated by quantitative PCR in colorectal mucosal biopsies.

Results and discussion: Data were available for 20 control participants and for 22 obese participants with matched pre- and postsurgery samples. Next Generation Sequencing revealed that compared with non-obese individuals, obese individuals showed differential expression of 112 microRNAs $(\mathrm{p}<0.05)$. Roux-en-Y gastric bypass, resulted in differential expression of 60 microRNAs, when compared with expression levels at baseline $(p<0.05)$. A total of 36 microRNAs differed significantly in both i) the obese with nonobese and ii) the pre- and post-surgery comparisons. Validation by quantitative PCR demonstrated that expression of miR-31, miR-215, miR-3196 and miR-4516 was significantly $(\mathrm{P}<0.05)$ higher in obese than in non-obese individuals. Weight loss, (mean $28.5 \mathrm{~kg}$ ) following Roux-en-Y gastric bypass, reduced expression of miR-31, miR-215 and miR-3196 significantly (P<0.05) to expression levels that were comparable with those in Controls. These differentially expressed microRNAs are implicated in pathways linked with inflammation, obesity and cancer.

Conclusion: The pattern of microRNA expression in macroscopically-normal human colorectal mucosa differed substantially between obese and non-obese individuals. However, six months after Roux-en-Y gastric bypass, the pattern of microRNA expression was similar to that in non-obese Controls. This suggests that surgically-induced weight loss may normalise microRNA expression in the human colorectal mucosa and so reduce CRC risk.

\section{Conflict of Interest}

There is no conflict of interest. 